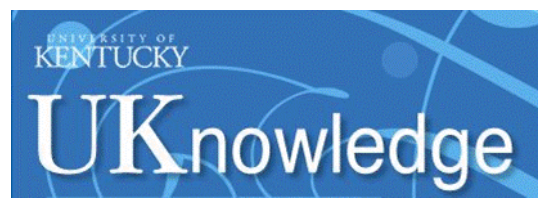

University of Kentucky

UKnowledge

$11-2019$

\title{
Las Dos Cosas versus Exclusive Breastfeeding: A Culturally and Linguistically Exploratory Intervention Study in Hispanic Mothers Living in Kentucky
}

\author{
Ana Maria Linares \\ University of Kentucky, am.linares@uky.edu \\ Diana Cartagena \\ Old Dominion University \\ Mary Kay Rayens \\ University of Kentucky, mkrayens@uky.edu
}

Follow this and additional works at: https://uknowledge.uky.edu/nursing_facpub

Part of the Nursing Commons, and the Pediatrics Commons

Right click to open a feedback form in a new tab to let us know how this document benefits you.

\section{Repository Citation}

Linares, Ana Maria; Cartagena, Diana; and Rayens, Mary Kay, "Las Dos Cosas versus Exclusive Breastfeeding: A Culturally and Linguistically Exploratory Intervention Study in Hispanic Mothers Living in Kentucky" (2019). Nursing Faculty Publications. 52.

https://uknowledge.uky.edu/nursing_facpub/52

This Article is brought to you for free and open access by the College of Nursing at UKnowledge. It has been accepted for inclusion in Nursing Faculty Publications by an authorized administrator of UKnowledge. For more information, please contact UKnowledge@lsv.uky.edu. 


\title{
Las Dos Cosas versus Exclusive Breastfeeding: A Culturally and Linguistically Exploratory Intervention Study in Hispanic Mothers Living in Kentucky
}

\author{
Digital Object Identifier (DOI) \\ https://doi.org/10.1016/j.pedhc.2019.07.009 \\ Notes/Citation Information \\ Published in Journal of Pediatric Health Care, v. 33, issue 6. \\ (C) 2019 by the National Association of Pediatric Nurse Practitioners \\ (c) 2019. This manuscript version is made available under the CC-BY-NC-ND 4.0 license \\ https://creativecommons.org/licenses/by-nc-nd/4.0/.
}

The document available for download is the authors' post-peer-review final draft of the article. 


\title{
"Las dos cosas" versus exclusive breastfeeding: A culturally and linguistically exploratory intervention study in Hispanic mothers living in Kentucky
}

\author{
ANA MARIA LINARES, DNS, RN, IBCLC [Visiting Professor], \\ College of Nursing, University of Kentucky, Lexington, KY. \\ Faculty of Health Science, Universidad de Tarapaca, Arica, Chile. \\ DIANA CARTAGENA, PHD, RN, CPNP, \\ School of Nursing, Old Dominion University, Norfolk, VA50 \\ MARY KAY RAYENS, PhD \\ College of Nursing and College of Public Health, University of Kentucky, Lexington, KY.
}

\section{Abstract}

Introduction.-Supplementation with formula feeding among infants of immigrant Hispanics that breastfeed is common. This phenomenon is known as las-dos-cosas. The purpose of this study was to assess the feasibility/effectiveness/acceptability of a culturally/linguistically intervention to promote exclusive breastfeeding (EBF) for the first six months.

Methods.-A sample of 39 Hispanic pregnant women were recruited and randomly assigned to Intervention ( $\mathrm{n}=20)$ and Control groups $(\mathrm{n}=19)$. The intervention incorporated a peer counselor/ professional support and mothers were followed from pregnancy to 6-months after birth.

Results.-After the intervention, the post-hoc comparison of the two treatment groups indicates that compared with the Control group, those in the Intervention group were over three times more likely to EBF their baby, when considering all four postpartum timepoints assessed (OR:3.1;

95\% CI: 1.1 to 8.7 ).

Corresponding author: Ana M. Linares, DNS, RN, IBCLC, 315 College of Nursing Building, University of Kentucky, Lexington, KY, 40536-0230, USA. Phone: 859-323-4626, Fax: 859-323-1057, am.linares@uky.edu.

Contributor Statement

A.M.L. developed the framework, implemented the study, and supervised the findings of this study. M.K.R. run the statistical analysis than were verified by A.M.L. and D.C. All authors discussed the results and wrote the final manuscript. The final manuscript was approved by all authors.

Publisher's Disclaimer: This is a PDF file of an unedited manuscript that has been accepted for publication. As a service to our customers we are providing this early version of the manuscript. The manuscript will undergo copyediting, typesetting, and review of the resulting proof before it is published in its final form. Please note that during the production process errors may be discovered which could affect the content, and all legal disclaimers that apply to the journal pertain.

Declaration of Conflicting Interests

The authors declared no potential conflicts of interest with respect to the research, authorship, and/or publication of this article.

Ethical Statement

This study was approved by the Medical Institutional Review Board (IRB) of the University of Kentucky. Additionally, a Certificate of Confidentiality was obtained from the National Institutes of Child Health and Human Development to assure privacy and protect participants' sensitive information on immigration status and/or mistrust in sharing their personal information. 
Conclusion.-This culturally/linguistically intervention contributed to increasing EBF duration in this sample of Hispanic mothers and significantly decreased formula supplementation at 6months post-birth.

\section{Keywords}

Exclusive Breastfeeding; Lactation Consultant; Peer Counselor; Breastfeeding intervention; Hispanics

\section{Introduction}

There are racial and ethnic disparities in breastfeeding (BF) duration and exclusivity impacting maternal and child health outcomes and cost (Bartick et al., 2017; Jones, Power, Queenan, \& Schulkin, 2015). Compared with non-Hispanic white children, Hispanic children have an increased risk of excess diseases attributable to suboptimal BF such as acute otitis media, gastrointestinal infection, and child death (Bartick et al., 2017).

Hispanic women who immigrate to the U.S. bring with them cultural traditions and healthcare practices from their native country that are protective behaviors, such as the culture of exclusive breastfeeding (EBF). However, researchers have identified increased use of formula feeding among low-income immigrant Hispanic women once in the US, referring to this phenomenon as "las dos costs" (both things), denoting the use of formula and breastmilk simultaneously (Bartick \& Reyes, 2012; Hernandez, 2006; Hohl, Thompson, Escareno, \& Duggan, 2016; Linares, Rayens, Dozier, Wiggins, \& Dignan, 2015; NommsenRivers, Chantry, Cohen, \& Dewey, 2010). Factors impacting the use of formula among Hispanic women include easy and affordable access to formula and misperceptions regarding the nutritional and health benefits and parental responsibilities pertaining to formula use (Bartick \& Reyes, 2012; Flores, Anchondo, Huang, Villanos, \& Finch, 2016; Hernandez, 2006). Mixed feeding not only jeopardizes milk production but also produces overfeeding that leads to childhood overweight and obesity, as well as increased risk of other diseases (Bartick et al., 2017; Cartagena, McGrath, \& Linares, 2018; Cartagena et al., 2014). These risks may be preventable with longer duration of EBF.

Few investigators have described tailored interventions targeting exclusively immigrant or first generation of Hispanic women. Most interventions targeting Hispanic mothers have been effective in increasing the initiation of BF; behavior that is known as usual in Hispanic women; but have had only modest impact on duration and exclusivity (Bunik et al., 2010; Howell, Bodnar-Deren, Balbierz, Parides, \& Bickell, 2014; Joshi, Amadi, Meza, Aguire, \& Wilhelm, 2016; Reeder, Joyce, Sibley, Arnold, \& Altindag, 2014; Washio et al., 2017). Lutenbacher et al. (2018) implemented a peer home visiting program and showed a significant effect on EBF for at least six weeks compared with the attentional matched control group. The strong belief among Hispanics that formula is a healthy choice and the best solution to BF difficulties appear to be the factors in the lack of successful interventions promoting duration of EBF (Bartick \& Reyes, 2012; Bunik et al., 2010; Chapman, 2010). In the absence of an effective early intervention to engage immigrant Hispanic mothers in sustained EBF, disparities in health risk behavior and associated diseases among Hispanic 
children and families are destined to continue escalating over the next decade, further exacerbating inequities in quality of life and health-care burden in this vulnerable population.

The purpose of this exploratory randomized clinical trial (RCT) study was to assess the feasibility, effectiveness, and acceptability of a culturally tailored intervention to promote $\mathrm{EBF}$ for the first six months. In particular, the aims were to evaluate the group differences trends in Intention to BF and BF knowledge during the prenatal period ( 2 assessments) and EBF status starting with discharge from the hospital and continuing for six months (4 assessments), as well as summarize acceptability as rated by those randomized to the intervention group.

\section{Methods}

This study used an exploratory longitudinal design, single site, RCT, and was approved by the Medical Institutional Review Board (IRB). After IRB approval, a Certificate of Confidentiality was obtained from the National Institutes of Child Health and Human Development to assure privacy and protect participants' sensitive information on immigration status and/or mistrust in sharing their personal information. This study was retrospectively registered in clinicaltrial.gov, number .

\section{Sampling}

A sample of 39 self-identified Hispanic pregnant women was recruited and randomly assigned to Intervention $(n=20)$ and Control (i.e., standard care) groups $(n=19)$. To ensure a relatively homogenous group, the inclusion criteria were: 1) self-identify as Immigrant Hispanic women; 2) pregnant at or beyond 30 weeks of gestation; 3) intention to at least try to breastfeed; 4) planning to deliver at a local birthing hospital; and 5) planning to remain in the area for at least 6 months after the birth of their child. There were no age limits for participation. To avoid potential complications with BF efforts, the exclusion criteria were: 1) prior or current participation in any study to enhance BF; 2) pregnant with twins; 3 ) history of breast surgery; 4) contraindication to BF (e.g., HIV-positive status, chronic therapy with medications incompatible with BF, alcohol dependence or other substance abuse); and 5) presumed or known congenital fetus defects. The CONSORT flow diagram for this study is presented in Figure 1 (Eldridge et al., 2016). Given the exploratory nature of this study, a priori power analysis was not conducted.

\section{Procedure}

Participants were recruited from a local primary health care clinic that provides care to most of the Hispanics in Central X. Women were approached in a waiting area reserved for pregnant patients by bilingual and bicultural trained research personnel, who explained the objectives of the study, checked for eligibility and invited them to participate in the study. Women who agreed to participate were scheduled for an appointment at their home or clinic to get the baseline information. Women participating signed a written informed consent and HIPAA forms, and younger of 18 years old signed the assent form, except if they were emancipated minors. Recruitment, informed consent documents, and interviews were 
administered in Spanish because all participants spoke Spanish as their primary language. Participants received their group assignment after the baseline interview was completed. Data collection was conducted by a trained research staff member who was blinded about participant randomization; blinding was maintained to decrease the potential for any discomfort the mother may have felt in responding to questions about infant feeding status if she was not BF her infant. Data were collected twice during pregnancy (at or past 30 and 37 weeks), medical chart review after the birth of the infant, and at 1-, 3- and 6-months following birth. Women participating in the study were compensated with a gift card for their time in each visit.

\section{Intervention}

The Principal Investigator (PI), a bilingual and bicultural International Board Certified Lactation Consultant (IBCLC), led the intervention. The Peer Counseling Training Platform from United States Department of Agriculture was used to train the Peer Counselor (PC), who was recruited from the local Hispanic community and who had two previous successful experiences of EBF her infants for six months. The PC completed 20 hours of training.

Mothers randomly assigned to the Intervention group were contacted by the PI and the trained bicultural PC to initiate the intervention. The content of the intervention was guided by concepts of the Behavior-Specific Cognitions and Affect variables within the Health Promotion Model (HPM) (Pender, Murdaugh, \& Parsons, 2002). These variables constitute a critical core for intervention and are considered to be of major motivational significance because they are subject to modification. Five HPM concepts were included for the development of the intervention: perceived benefits to action, perceived barriers to action, perceived self-efficacy, interpersonal influence, and situational influence (Figure 2). Key components of the intervention were: First, informational material was prepared with the goals of 1) raising consciousness - through seeking and processing awareness of benefits of adopting a healthy behavior (i.e., EBF for six months, delaying introduction of solid food) or discontinuing a risky behavior (e.g., supplement with formula, infant solid food before six months); 2) anticipating barriers to EBF-perceptions concerning the unavailability, inconvenience, difficulty, or time-consuming nature of the action; and 3) promoting selfefficacy. BF self-efficacy was acknowledged and referred to beliefs about being able to carry out progressively more demanding levels of EBF, and to overcome barriers to engage in the behavior (empower). Second, individual home-visit sessions were designed to increase trust to enhance the benefits of change (e.g., reinforcing motivation, benefit and positive outcomes derived from the behavior), and self-efficacy to control interpersonal and situational influences. Third, a commitment to a plan of action was developed based on adapting (including cultural and linguistic adaptation) My Action Plan for BF (MAP) from the California Nutrition and Physical Activity Guidelines for Adolescents (California Department of Public Health, 2015). The intensive peer counselor/professional support (1-2 prenatal visits, one in-hospital visit, two home postpartum visits, and pre/post-natal followup phone calls as needed) was conducted individually with each mother until six months after the birth of the infant (Table 1). In case of failure to meet an encounter either for the data collection and/or intervention (e.g., preterm birth, lack of time, work, sickness, etc.), participants were not excluded from the study. The IBCLC, PCs, and research staff remained 
flexible to the participants' schedule in order to acquire complete data and arrange the intervention sessions.

Women in the usual care group received the regular education on BF that was given to all women during their prenatal care visit in the clinic. Additionally, women from both groups gave birth in a "Baby Friendly Hospital" that allowed them to receive support from a clinical IBCLC from the birthing hospital. Women in the control group did not have any contact with the IBCLC/PC study team.

\section{Measures}

Demographic and Personal Characteristics.-Once participants were screened for eligibility and enrolled in the study, demographic characteristics in the baseline survey included age (in years) and a series of yes/no items, including whether they lived with their partner or husband, were employed, had health insurance, and enrolled in the Women, Infant, and Children Program (WIC). Mode of delivery was recorded from the medical record following the birth.

Previous Experience with $B F$ was measured with a single question that assessed if the mother has ever had anyone close to her breastfeed (yes/no response option); for those who answered 'yes,' there was a follow-up item asking who this person was.

Acculturation.-The 7-item short version of the Hispanic Acculturation Scale (Marin, Sabogal, Marin, Otero-Sabogal, \& Perez-Stable, 1987) assessed the likelihood of using Spanish or English in different situations. Responses are rated in a five-point choice: only Spanish; Spanish better than English; both equally; English better than Spanish; and only English. The responses are summed, with the total score ranging from 5 to 35, with higher scores indicating a greater degree of acculturation. Cronbach's alpha was 0.91 for this sample.

Infant Feeding Plan and Potential BF Goal were assessed at baseline and again before the birth of the infant using the Infant Feeding Intentions Scale, Spanish version (IFI) (Nommsen-Rivers, Cohen, Chantry, \& Dewey, 2010). This scale has five items, with response options ranging from $0=$ 'Very much disagree' to $4=$ 'Very much agree.' The total score is obtained by averaging the first two items (which include planning about giving BF a try and another item that is reverse-coded as it is an endorsement of planning to use formula) and adding the remaining three items to this mean. The range of scores is from 0 to 16 , with higher scores indicating a stronger intention to EBF for six months. Cronbach's alpha for this sample was 0.87 .

BF Knowledge was assessed at baseline and again before the birth of the infant using an adapted Spanish version of the BF Knowledge Questionnaire (Wambach et al., 2011). The questionnaire has 25 items, with response options $1=$ True, and $0=$ False. The total score is obtained by first reversing eight of the items by assigning a value of 1 for each correct answer of 'False' and then adding all the items. The range of scores is from 0 to 25 , with higher scores indicating greater knowledge of BF. Kuder-Richardson 20 for this sample was 0.72 . 
Infant Feeding Status was determined at discharge from the hospital immediately following birth via medical chart review from reports in the infant's chart, and compared for accuracy with mother's chart notes from the physician, lactation consultant, nurse or another clinician. Follow-up infant feeding status at each encounter post-discharge (1,3, and 6 months), was assessed via the mother's report; First the mother was asked to choose the practice that most closely resembles how she was feeding her infant with possible options of EBF, predominant breast milk; mixed feeding; predominant formula milk; and exclusive formula milk (Thulier, 2010; World Health Organization, 2017). Then, the mother was also asked, 'Does your baby receive water, juice, or any solids?' If the mother agreed that the infant is receiving additional food, mothers were asked when they introduced the food to their child. Only those who replied that were only given breastmilk without any additional food were recorded as EBF for feeding status.

Acceptability of the Intervention was assessed at the end of the study for the participants that were assigned to the intervention group. Only participants who completed the study were evaluated using this instrument. This scale has 5-items assessing the work of PC and IBCLC, and a woman's feeling of satisfaction with the intervention experience. Response options ranging from $1=$ "very dissatisfied" to $5=$ "very satisfied." The range of scores is from 5 to 25 , with higher scores indicating greater acceptability of the intervention.

\section{Data Analysis}

Study variables were summarized using means and standard deviations or frequency distributions. Comparisons of the intervention and control groups, or between those who completed the study and those who dropped out, were accomplished using two-sample ttests, chi-square tests of association, or Fisher's exact tests as appropriate. The longitudinal group comparisons of Intention to BF and BF Knowledge were done using mixed modeling for repeated measures. The initial model contained the main effects of group (Intervention vs. Control), time (2 timepoints during prenatal period), and their interaction.

Similarly, the group comparison of EBF status over the four timepoints (discharge and 1,3, and 6 months) was accomplished using generalized estimating equations (GEE) modeling given the binary outcome. In this model, we included baseline Intention to $B F$ as a covariate because although the group difference in intention at baseline was not significant at the specified alpha level, there was a difference in means between the groups at the 0.1 level, with the Intervention group having higher average Intention to $B F$ scores than Controls. In addition to the covariate, the initial GEE model included the main effects of treatment (Intervention vs. Control), time (4 timepoints) as well as their interaction. In all three final longitudinal models (i.e., for Intention, Knowledge, and EBF status), the treatment by time interaction was not included since this term was not significant in any of the models. Data analysis was conducted using SAS, v 9.4; an alpha of 0.05 was used.

\section{Results}

The average age of the 39 participants was 25.4 years $(S D=5.9)$, with a range from 13 to 37. Most lived with their partner (71.8\%), were not employed outside the home (71.8\%), did not have medical insurance (66.7\%), and were registered (66.7\%) in WIC. The mode of 
delivery was most often vaginal (76.9\%), and the same percentage reported that someone close to them had breastfed previously. Acculturation scores were typically relatively modest compared with the maximum possible score of 35, with a mean of $11.7(S D=5.9)$ and scores ranging from 7-24. In the full sample (comprising both Intervention and Control participants), the Intention to $B F$ score was relatively high at enrollment: the mean was 12.3 ( $S D=3.6$ ), with a range from $0-16 . B F$ Knowledge scores were also relatively high, with an average of $19.5(S D=3.6)$, and scores ranged from 10 to 25 . As shown in Table 2, there were no significant differences between the two treatment groups on any of these demographic, clinical, or personal variables. Only baseline Intention to $B F$ demonstrated a marginal difference $(p=.1)$, with a higher average score in the Intervention group relative to Control.

The comparisons of those who completed the study $(n=29)$ to those who dropped out by six months $(n=10)$ on these baseline characteristics suggested that completers and dropouts were comparable as well. For each of the variables listed in Table 2, there was no difference between these two subgroups ( $p>.2$ for each comparison).

The repeated measures model for Intention to $B F$ was significant overall (Likelihood ratio $\left.\chi^{2}=29.0, p<.001\right)$. While the enrollment Intention to $B F$ score did not differ significantly between the two treatment groups ( $p=.10$, see Table 2$)$, the group main effect in the repeated measures model was significant $(\mathrm{F}=5.0, p=.031)$; the Intervention group had higher average scores during the prenatal period than the Control group. Averaged over the two prenatal assessments, the mean Intention to $B F$ scores for the Intervention and Control groups were 13.6 and 11.1, respectively. The main effect of Time was not significant in this model $(p=.68)$, suggesting the degree of change over time was not significant for the two groups combined. The repeated measures model for the BF Knowledge was not significant overall (Likelihood ratio $\chi^{2}=2.7, p .10$ ). Although the mean for the Intervention group exceeded that of the Control, this observed difference was not great enough to lead to overall model significance, so the group comparison is not warranted.

BF initiation at hospital stay was high in both groups, with $100 \%$ and $90 \%$ in the Intervention vs. Control group respectively. However, for the more stringent standard of EBF, $45 \%$ of mothers in the Intervention group and $21 \%$ of those in the Control group were in the EBF group during hospitalization following the birth. More mothers in the Control group initiated "las dos cosas" or BF and supplementing with formula (68\%) compare with the Intervention group (55\%). Since the baseline mean for Intention to BF was somewhat higher in the Intervention group relative to the Control, albeit not statistically significant, this was used as control variable in the longitudinal analysis of EBF status. The final model for the repeated measures assessment of group and time differences in EBF status, controlling for baseline Intention to $B F$, indicated that the Group main effect was significant $\left(\chi^{2}=4.3, p=.038\right)$, and was the main effect of Time $\left(\chi^{2}=10.6, p=.014\right)$. The post-hoc comparison of the two treatment groups indicates that compared with the Control group, those in the Intervention group were three times more likely to EBF their baby (odds ratio [OR]: 3.1), when considering all four postpartum timepoints (95\% confidence interval for the OR: 1.1 to 8.7), and controlling for baseline Intention to BF. The significance of the main effect of Time corresponds to the decrease in EBF prevalence over time, especially 
between three and six months. While the lack of a significant interaction between Group and Time precludes the post-hoc comparison of EBF status at each timepoint; Figure 3 displays the frequency of EBF mothers at each timepoint, with a clear pattern of greater percent of EBF success in the Intervention group regardless of timepoint. In the Intervention group in particular, the greater decline in EBF prevalence between 3 to 6 months was largely due to the introduction of solid foods during months 4-5 at the advice of their care providers: while half of Intervention group participants were still using breastmilk as the only liquid food source at 6 months, $71 \%$ of these mothers had introduced solid food prior to this assessment and so were not considered EBF at 6 months for this analysis.

Although the difference in retention was not significant between the groups, there was a slightly higher retention rate at six months among those in the Control group relative to the Intervention group (79\% vs. 70\%, respectively; Fisher's exact $p=.72$ ). In light of this difference, we also considered a sensitivity analysis of the GEE model, in which we assumed that all missing EBF status observations were 'no' for this variable, for mothers in both groups. This is a conservative analysis since it favors the null hypothesis of no group difference in EBF status. The sensitivity model, also with Intention to BF included as a covariate, was relatively consistent with the GEE model described above. In particular, the main effect of Group was significant $\left(\chi^{2}=4.2, p=.040\right)$, and the Time main effect was as well $\left(\chi^{2}=12.8, p=.0051\right)$. The post-hoc comparison of the Treatment main effect indicates that compared with the Control group, those in the Intervention group were three times (OR = 3.0) times more likely to EBF their baby, when considering all four post-birth timepoints ( $95 \%$ confidence interval for the OR: 1.1 to 8.8 ). The consistency of findings from the original model and sensitivity analysis underscores the stability of this evaluation, even with $26 \%$ of the participants not completing the study.

\section{Intervention.}

Seventy percent $(n=14)$ of participants in the intervention group received two prenatal home visits as was established by the intervention; while $30 \%(n=6)$ only got one home visit. Similarly, the hospital visit was received by $70 \%$ of participants in the intervention group. IBCLC visited $75 \%(\mathrm{n}=15)$ of mothers at their house while PC was able to complete the home visit to $45 \%$ ( $n=9)$ of mothers. Most of the mothers (60\%) got at least two follow-up calls before delivery, while the rest got one. During postpartum, most of the mothers (75\%) got two follow-up calls. No answer to the phone calls and unreachable were the causes why some sessions were not completed. Attrition in the intervention group (30\%) was mostly during the six months follow up phone call (Figure 1).

The average score in Acceptability of the intervention among participants in the intervention group and completed the study was 22.7 points ( $\mathrm{SD}=2.5$ ), with a range from 17 to 25 . Most of the participants that completed the intervention (93\%) were satisfied or very satisfied with the intervention. All except four participants were very satisfied (71\%) with the support given by the PC, and also all except two participants were satisfied to very satisfied (86\%) with the intervention of the IBCLC. 


\section{Discussion}

In this RCT exploratory study, we aimed to assess the effectiveness of a culturally/ linguistically tailored intervention to promote EBF for the first six months in a sample of Hispanics mothers living in X. Specifically, we aimed to evaluate the group differences (Intervention vs. Control) in Intention to BF and BF Knowledge during the prenatal period and EBF status starting with discharge from the hospital and continuing for six months. The treatment groups (Intervention vs. Control) were comparable at baseline, and there was not a difference in baseline characteristics between dropouts and completers.

Our findings indicate that most of the women in both groups reported a high intention to initiate BF and were planning to EBF their infants. However, when both Intention to BF scores collected during pregnancy were included in a longitudinal model, the Intervention group had an average score that was 2.5 points higher than the Control group across the two timepoints, which occurred after at least one face to face intervention session was received by the Intervention group, and this difference was significant. This suggests that prenatal support by the bilingual/cultural trained PC and IBCLC had a positive effect. Intention to BF has been described as one of the modifiable behaviors to increase adherence to EBF (Meedya, Fahy, \& Kable, 2010). The prenatal portion of the intervention included phone contact with the IBCLC and PC home visiting with discussions of BF educational modules, anticipatory guidance on the management of barriers, solving problems, and enhancing selfefficacy on BF. We believe the personalized education and home visiting is highly recommended for this population that most of the time report lack of time or transportation issues to visit the clinic or another place (Kaiser Family Foundation, 2018). Additionally, building of trust between the IBCLC and PC with the participant was encouraged with the individualized culturally/linguistically tailored support and home visiting (Park, Schwei, Xiong, \& Jacobs, 2018). The Department of Human and Health Services has reported the effectivity of implementing home visiting in minority groups (Sama-Miller et al., 2017).

The group effect was significant for EBF status - combining across the four post-birth assessments, and controlling for baseline Intention to $B F$, those in the Intervention group were three times more likely to EBF, relative to Control participants. This group effect was maintained even when missing EBF status observations were replaced with 'no' values. It has been demonstrated that Hispanic mothers are typically willing to initiate BF (Hohl et al., 2016; Linares et al., 2015) as was verified in our study with almost $95 \%$ of the mothers participating in our study (including both Control and Intervention groups) initiating BF at hospital stay. However, in the Control group, many mothers requested formula during the hospitalization (las dos cosas). This phenomenon has been highly discussed from others authors (Bartick \& Reyes, 2012; Hohl et al., 2016; Nommsen-Rivers, Chantry, et al., 2010) and seems to be the factor that jeopardizes the exclusivity of BF. Additionally, some researchers in South America have warned about the declining of EBF in their countries with a switch to "las dos cosas" (Boccolini, Boccolini, Monteiro, Venancio, \& Giugliani, 2017; Gonzalez de Cosio, Escobar-Zaragoza, Gonzalez-Castell, \& Rivera-Dommarco, 2013). It seems like Hispanic mothers want to breastfeed their infants but at the same time supplement with formula, which increases the risk of overfeeding their infants (Cartagena et al., 2014). There is evidence that EBF children that are introduced to solids right after six 
months and continued to receive breastmilk ( $\geq 12$ months) are less likely to become overweight/obese (Papoutsou et al., 2018). Hispanic infants are at high risk of childhood obesity and this risk factor is presented at an early stage as was reported by the National Health and Nutrition Examination Survey (2014), where the prevalence of high weight for recumbent length ( $₫ 95$ th percentile) among infant and toddlers from birth to 2 years was 6.6\% in Whites, $8.4 \%$ in Blacks, and 9.4\% in Hispanics (Ogden, Carroll, Kit, \& Flegal, 2014). Promotion of EBF for six months in Hispanic children potentially decreases the high risk of childhood obesity. This intervention was able to provide mothers with the support that was required to avoid supplementation with formula from the infant's birth until at least three months of age. However, many mothers in the Intervention group who had maintained EBF until this point were advised by their health care provider to supplement with juice and solid food at ages 4-5 months, thus ending EBF prior to 6 months. Consistent with this phenomenon, the largest decline in EBF prevalence in the Intervention group was between three and six months. The American Academy of Pediatrics (AAP) and World Health Organization (WHO) recommend EBF for a minimum of six months followed by continued BF for a minimum of one year as complementary foods are introduced (AAP, 2012; WHO, 2017); however, as was observed in our study many mothers were advised to initiate the introduction of food before the recommended time.

\section{Implication for Practices}

A common infant feeding option for Hispanics is "las dos cosas." Researchers have observed that even this behavior is common in newly immigrant, low-income Hispanic women once in the US. It is known that most of the immigrant Hispanic mothers are going to initiate breastfeeding, however, they learn from their peers that formula is acceptable and could be easily available from the Women, Infants, and Children (WIC) program, which makes combination feeding, or even discontinuation of breastfeeding altogether, possible (Hernandez, 2006). Our study showed that mothers that received the intense peer counselor/ professional lactation support were able to overcome the misconception that "las dos cosas" is a healthy choice, and they were more likely to keep their infant with EBF for a longer time. An important implication for practice is that the cultural/linguistically appropriate intervention, which included home visits by the PC and IBCLC to support the Hispanic mothers were able to produce the behavioral change needed. It is evidence that when mothers are from a cultural background different from the health provider, additional challenges are described including language, different cultural beliefs, mistrust, and limited illiteracy (Lutenbacher et al., 2018; Park et al., 2018; Sobel \& Metzler Sawin, 2016). Therefore, cultural sensitivity is encouraged in health providers to be responsive to the health beliefs, practices, cultural and linguistic needs of diverse patients with the goal to help bring positive health outcomes (Park et al., 2018).

Another important implication is that health providers need to evaluate the benefit of maintaining a child with EBF and encourage mothers that are EBF to do so until six months. Our study showed that many mothers that were EBF incorporated solid food or other foods following the recommendation of their infant's health providers. A Cochrane review showed that for infants at four to six months, there was no evidence of benefit from additional foods earlier than six months (Becker \& Remmington, 2014; Smith \& Becker, 2016). Further 
intervention with health providers to promote current evidence-based practices recommendations on infant feeding is warranted.

\section{Strengths and Limitations}

The primary strength of this exploratory study was that the randomization yielded two treatment groups that were statistically comparable in every demographic, clinical, and personal characteristic included. Knowing the high probability that Hispanics have to leave an area to move for better opportunities, a 74\% retention at six months postpartum was considered relatively high. Further, there were no demographic differences between those who completed the study and those who dropped out. This latter observation helps bolster the use of a repeated measures modeling strategy that allows for the inclusion of participants in the analysis even if they have missing data, if it can be assumed that the data are missing at random. An additional strength is the finding that the significant group difference for EBF status in the postpartum period is maintained even if all missing values are replaced with EBF status equal to 'no.' The main limitation to this exploratory study is the sample size, but we were able to discern group differences in key outcomes, including Intention to $B F$ and $B F$ Knowledge, both assessed during pregnancy, and EBF status in the postpartum period. An additional limitation is that those randomized to the Intervention group had a slightly higher Intention to $B F$ at baseline $(p=.10)$, but this concern is mitigated since the group difference in EBF status during the postpartum 6-month period is maintained with Intention to $B F$ included as a covariate. These findings underscore the impetus for a larger study to further evaluate this culturally/linguistically tailored intervention for the promotion of EBF in this population. Finally, not all variables that affect breastfeeding behavior in Hispanics are included in this analysis. However, we prioritized the potentially modifiable factors that have the potential to be affected by an innovative, culturally, and linguistically appropriate intervention.

\section{Conclusions}

This novel, culturally/linguistically tailored intervention approach was highly acceptable to participants, contributed to increasing EBF duration in this sample of Hispanic mothers, and significantly decreased formula supplementation, something that has been rarely reported at six months postpartum in previous studies. The potential of this intervention is important in this at-risk population, particularly since government agencies have warned of the risk of formula supplementation at early stage, which is a detriment to both mothers and infants, compared with the health benefits of EBF. Future work will evaluate if any specific component of the intervention was significantly associated with duration of EBF or if the intervention as a whole help promotes extended EBF.

\section{Funding}

The project described was supported by the NIH National Center for Advancing Translational Sciences through grant number UL1TR000117 and UL1TR001998. The content is solely the responsibility of the authors and does not necessarily represent the official views of the NIH. 


\section{References}

American Academy of Pediatrics. (2012). Breastfeeding and the use of human milk. Pediatrics, 129(3), e827-841. doi:10.1542/peds.2011-3552 [PubMed: 22371471]

Bartick MC, Jegier BJ, Green BD, Schwarz EB, Reinhold AG, \& Stuebe AM (2017). Disparities in breastfeeding: Impact on maternal and child health outcomes and costs. Journal of Pediatrics, 181, 49-55.e46. doi:10.1016/j.jpeds.2016.10.028 [PubMed: 27837954]

Bartick M, \& Reyes C (2012). Las dos cosas: an analysis of attitudes of Latina women on nonexclusive breastfeeding. Breastfeeding Medicine, 7(1), 19-24. doi:10.1089/bfm.2011.0039 [PubMed: 22007765]

Becker GE, \& Remmington T (2014). Early additional food and fluids for healthy breastfed full-term infants. Cochrane Database Systematic Review (11), Cd006462. doi: 10.1002/14651858.CD006462.pub3

Boccolini CS, Boccolini PMM, Monteiro FR, Venancio SI, \& Giugliani ERJ (2017). Breastfeeding indicators trends in Brazil for three decades. Revista de Saude Publica, 51, 108. doi:10.11606/ s1518-8787.2017051000029 [PubMed: 29166437]

Bunik M, Shobe P, O'Connor ME, Beaty B, Langendoerfer S, Crane L, \& Kempe A (2010). Are 2 weeks of daily breastfeeding support insufficient to overcome the influences of formula? Academic Pediatrics, 10(1), 21-28. doi:10.1016/j.acap.2009.09.014 [PubMed: 20129478]

California Department of Public Health. (2015). California Nutrition and Physical Activity Guidelines for Adolescents. https://www.cdph.ca.gov/Programs/CFH/DMCAH/CDPH\%20Document $\%$ 20Library/NUPA/NUPA-Guidelines-Adolescents.pdf

Cartagena D, McGrath JM, \& Linares AM (2018). Associations between introduction of ageinappropriate foods and early eating environments in low-socioeconomic Hispanic infants. Journal Pediatric Health Care, 32(2), e27-e36. doi:10.1016/j.pedhc.2017.09.005

Cartagena DC, Ameringer SW, McGrath J, Jallo N, Masho SW, \& Myers BJ (2014). Factors contributing to infant overfeeding with Hispanic mothers. Journal Obstetrics Gynecology Neonatal Nurses, 43(2), 139-159. doi:10.1111/1552-6909.12279

Chapman DJ (2010). Exploring breastfeeding ambivalence among low-income, minority women. Journal of Human Lactation, 26(1), 82-83. doi:10.1177/0890334409355773 [PubMed: 20150336]

Eldridge SM, Chan CL, Campbell MJ, Bond CM, Hopewell S, Thabane L, \& Lancaster GA (2016). CONSORT 2010 statement: extension to randomized pilot and feasibility trials. Pilot Feasibility Study, 2, 64. doi:10.1186/s40814-016-0105-8

Flores A, Anchondo I, Huang C, Villanos M, \& Finch C (2016). "Las dos cosas," or why Mexican American mothers breast-feed, but not for long. Southern Medical Journal, 109(1), 42-50. doi: 10.14423/smj.0000000000000400 [PubMed: 26741873]

Gonzalez de Cosio T, Escobar-Zaragoza L, Gonzalez-Castell LD, \& Rivera-Dommarco JA (2013). Infant feeding practices and deterioration of breastfeeding in Mexico. Salud Publica Mexico, 55 Suppl 2, S170-179.

Hernandez IF (2006). Promoting exclusive breastfeeding for Hispanic women. MCN American Journal of Maternal Child Nursing, 31(5), 318-324. [PubMed: 17013073]

Hohl S, Thompson B, Escareno M, \& Duggan C (2016). Cultural norms in conflict: Breastfeeding among Hispanic immigrants in rural Washington state. Maternal Child Health Journal, 20(7), 1549-1557. doi:10.1007/s10995-016-1954-8 [PubMed: 27021068]

Howell EA, Bodnar-Deren S, Balbierz A, Parides M, \& Bickell N (2014). An intervention to extend breastfeeding among black and Latina mothers after delivery. American Journal Obstetrics Gynecology, 210(3), 239.e231-235. doi:10.1016/j.ajog.2013.11.028

Jones KM, Power ML, Queenan JT, \& Schulkin J (2015). Racial and ethnic disparities in breastfeeding. Breastfeeding Medicine, 10(4), 186-196. doi:10.1089/bfm.2014.0152 [PubMed: 25831234]

Joshi A, Amadi C, Meza J, Aguire T, \& Wilhelm S (2016). Evaluation of a computer-based bilingual breastfeeding educational program on breastfeeding knowledge, self-efficacy and intent to breastfeed among rural Hispanic women. International Journal Medical Informatics, 91, 10-19. doi:10.1016/j.ijmedinf.2016.04.001 
Kaiser Family Foundation. (2018). Health and health care for Hispanics in the United States. KFF KFF Website. Retrieved from https://www.kff.org/infographic/health-and-health-care-for-hispanics-inthe-united-states/

Linares AM, Rayens MK, Dozier A, Wiggins A, \& Dignan MB (2015). Factors influencing exclusive breastfeeding at 4 months postpartum in a sample of urban Hispanic mothers in Kentucky. Journal Human Lactation, 31(2), 307-314. doi:10.1177/0890334414565711

Lutenbacher M, Elkins T, Dietrich MS, \& Riggs A (2018). The efficacy of using peer mentors to improve maternal and infant health outcomes in Hispanic families: Findings from a randomized clinical trial. Maternal \& Child Health Journal, 22(Suppl 1), 92-104. doi:10.1007/ s10995-018-2532-z

Marin G, Sabogal F, Marin BV, Otero-Sabogal R, \& Perez-Stable EJ (1987). Development of a short acculturation scale for Hispanics. Hispanic Journal of Behavioral Sciences, 9 (2), 183-205

Meedya S, Fahy K, \& Kable A (2010). Factors that positively influence breastfeeding duration to 6 months: a literature review. Women and Birth, 23(4), 135-145. doi:10.1016/j.wombi.2010.02.002 [PubMed: 20299299]

Nommsen-Rivers LA, Chantry CJ, Cohen RJ, \& Dewey KG (2010). Comfort with the idea of formula feeding helps explain ethnic disparity in breastfeeding intentions among expectant first-time mothers. Breastfeeding Medicine, 5(1), 25-33. doi:10.1089/bfm.2009.0052 [PubMed: 20043707]

Nommsen-Rivers LA, Cohen RJ, Chantry CJ, \& Dewey KG (2010). The infant feeding intentions scale demonstrates construct validity and comparability in quantifying maternal breastfeeding intentions across multiple ethnic groups. Maternal Child Nutrition, 6(3), 220-227. doi:10.1111/j. 1740-8709.2009.00213.x [PubMed: 20929494]

Ogden CL, Carroll MD, Kit BK, \& Flegal KM (2014). Prevalence of childhood and adult obesity in the united states, 2011-2012. JAMA, 311(8), 806-814. doi:10.1001/jama.2014.732 [PubMed: 24570244]

Papoutsou S, Savva SC, Hunsberger M, Jilani H, Michels N, Ahrens W, ... Hadjigeorgiou C. (2018). Timing of solid food introduction and association with later childhood overweight and obesity: The IDEFICS study. Maternal Child Nutrition, 14(1). doi:10.1111/mcn.12471

Park L, Schwei RJ, Xiong P, \& Jacobs EA (2018). Addressing cultural determinants of health for Latino and Hmong patients with limited English proficiency: Practical strategies to reduce health disparities. Journal of Racial Ethnic Health Disparities, 5(3), 536-544. doi:10.1007/ s40615-017-0396-3 [PubMed: 28791616]

Pender N, Murdaugh C, \& Parsons M (2002). Health promotion in nursing practice: New Jersey, USA: Upper Saddle River

Reeder JA, Joyce T, Sibley K, Arnold D, \& Altindag O (2014). Telephone peer counseling of breastfeeding among WIC participants: a randomized controlled trial. Pediatrics, 134(3), e700709. doi:10.1542/peds.2013-4146 [PubMed: 25092936]

Sama-Miller E, Akers L, Mraz-Esposito A, Zukiewicz M, Avellar S, Paulsell D, \& Grosso PD (2017). Home visiting evidence of effectiveness review. Retrieved from Washington, DC: https:// homvee.acf.hhs.gov/homvee_executive_summary_august_2017_final_508_compliant.pdf Lactation, 25(4), 404-411. doi:10.1177/0890334409337308

Smith HA, \& Becker GE (2016). Early additional food and fluids for healthy breastfed full-term infants. Cochrane Database Syst Rev(8), Cd006462. doi:10.1002/14651858.CD006462.pub4

Sobel LL, \& Metzler Sawin E (2016). Guiding the process of culturally competent care with Hispanic patients: A grounded theory study. Journal of Transcultural Nursing, 27(3), 226-232. doi: 10.1177/1043659614558452 [PubMed: 25409673]

Thulier D (2010). A call for clarity in infant breast and bottle-feeding definitions for research. Journal of Obstetric, Gynecologic, \& Neonatal Nursing, 39(6), 627-634. doi:10.1111/j. 1552-6909.2010.01197.x

Wambach KA, Aaronson L, Breedlove G, Domian E, Rojjanasrirat W, \& Yeh HW (2011). A randomized controlled trial of breastfeeding support and education for adolescent mothers. Western Journal Nursing Research, 33(4), 486-505. doi: 410.1177/0193945910380408. Epub 0193945910382010 Sep 0193945910380427. 
Washio Y, Humphreys M, Colchado E, Sierra-Ortiz M, Zhang Z, Collins BN, ... Kirby KC (2017). Incentive-based intervention to maintain breastfeeding among low-income Puerto Rican mothers. Pediatrics, 139(3). doi:10.1542/peds.2016-3119

World Health Organization. (2017). Nutrition exclusive breastfeeding. Retrieved from http:// www.who.int/nutrition/topics/exclusive_breastfeeding/en/ 
Assersed for eligibility ( $(\mathrm{w}=185)$

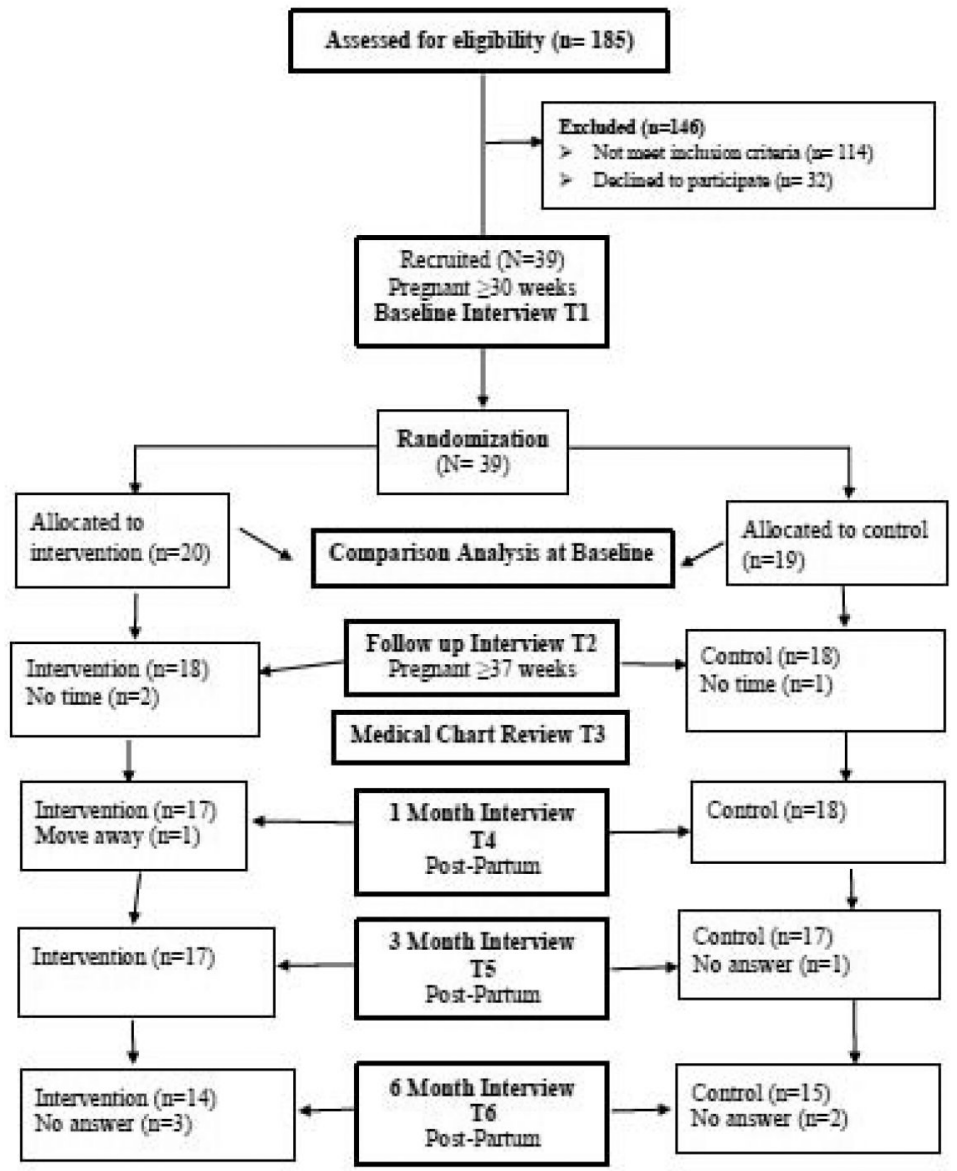

Figure 1.

CONSOR flow diagram pilot study (Eldridge et al, 2016) 


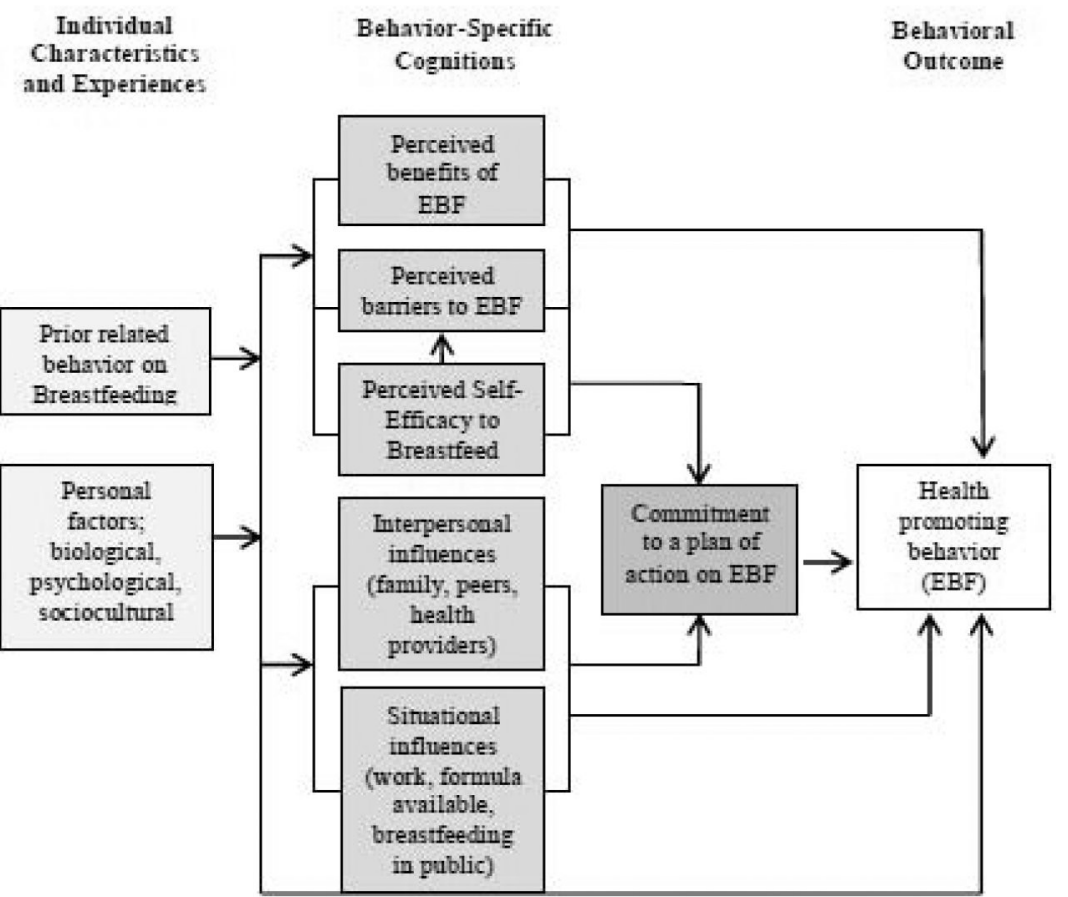

Figure 2.

Promotion of EBF in Hispanics Women in Kentucky. Adapted from the Health Promotion Model (Pender et al., 2002). 


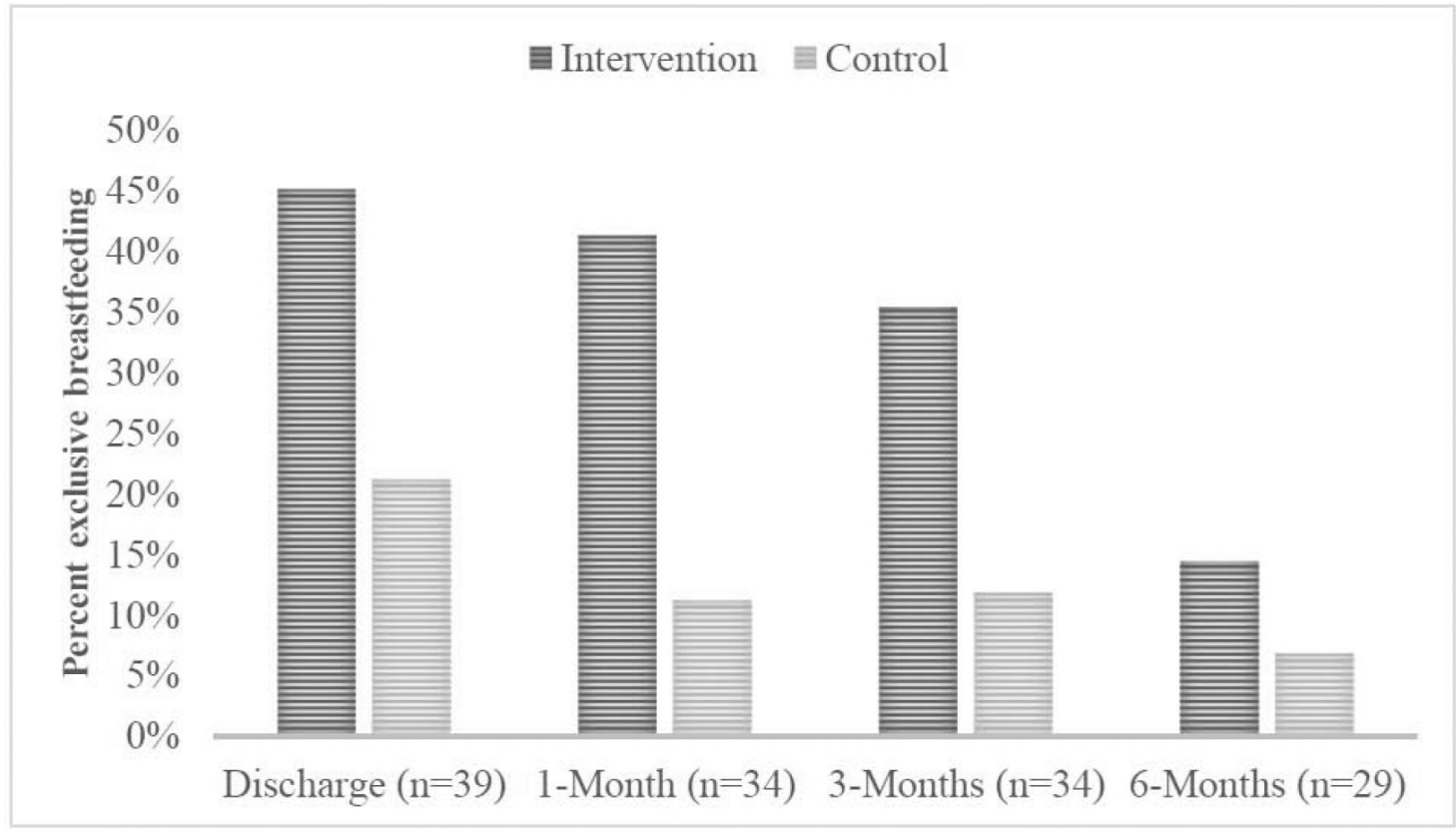

Figure 3.

Frequency of EBF in each group over time $(\mathrm{N}=39)$ 
Table 1:

Description of Sessions for Intervention Participants

Session
2 Prenatal home visit sessions (30-40
minutes each) by PC

Prenatal Follow-up phone calls (10-15 minutes each) by a PC

\section{Activities *}

Participant was oriented to the purpose of the intervention; collaboration was established between peer counselor and participant. Discussion of participant's past experiences with breastfeeding was initiated. Intervention was explained, including discussion of the benefits of adopting a healthy behavior (e.g., exclusive breastfeeding (EBF) for six months, delay introducing formula and/or solid until after six months old). Infant feeding and qualms about mixed feeding were described and discussed (las dos cosas), and barriers to EBF and self-efficacy were assessed. A general review of provided binder material: benefits of EBF, breastfeeding logistics, avoidance of pacifiers, feeding cues, breastfeeding video review. My Action Plan to Breastfeed was described, and participant settled her own goals.

My Action Plan to Breastfeed was reviewed, and participant commitments to breastfeeding goals were reinforced.

Barriers to EBF were reviewed, including perceptions concerning the lack of availability, inconvenience, difficulty, or time-consuming nature of the future action; and promoting selfefficacy with problem-solving.

Participant was encouraged to study program materials and was asked about any concerns associated with breastfeeding goals. Strategies available to accomplish her breastfeeding goals were discussed. All questions were answered. Follow-up calls were done every week until the birth of the infant.

Hospital Visit (20-30 minutes) by PC.

Assessment of infant feeding pattern. Observation of breastfeeding techniques with hands-on assistance when was needed. Discussion of techniques, feeding cues, demand feeding, and other support essential to maintain EBF, anticipate barriers, problem-solving, and self-efficacy. All questions were answered. Praised for her commitment to initiate breastfeeding was included.

\section{Postpartum \\ home-visit (40-60 minutes) at $\pm 2-3$ \\ days after discharge by an IBCLC}

\author{
1 Postpartum \\ home-visit (30-40 minutes) at 7-10 \\ days after discharge by $\mathrm{PC}$.
}

Postpartum phone calls (10-20 minutes each) at least once a month for 6 months by PC.
Assessment of infant feeding pattern. Observation of breastfeeding techniques with hands-on assistance if needed. Discuss techniques, feeding cues, demand feeding, and other issues needed to maintain the exclusivity of breastfeeding, anticipate barriers, share problem-solving strategies, and encourage self-efficacy. If needed, provide supplemental breastfeeding devices as needed (e.g., nipple shields, manual breast pump, nipple cream). Anticipatory guidance was given and all questions answered.

Lactation consultant followed-up with a phone call as needed. A second visit was scheduled when needed.

Home visit by PC to follow-up and encourage the mother in her commitment to EBF her infant. Guidance given on problems/concerns and questions were answered. Motivation and self-efficacy were promoted.

Praised the mother for her efforts in taking care of her infant. Anticipatory guidance based on the age of the infant was given. Mother was encouraged to call the IBCLC if any concerns arise.

We asked the mother to describe her experience with infant feeding.

We asked about problems/concerns and answered questions. Promoted motivation and selfefficacy.

Praised the mother for her effort in taking care of her infant. Anticipatory guidance based on the age of the infant. Encouraged the mother to call the peer counselor and/or lactation consultant if any concerns arise. Initial follow-up phone calls were no later than 2 weeks after the birth of the infant. Repeated phone calls at least once a month for 6 months. Refered to IBCLC when needed.

* For further information visit ClinicalTrial.gov

$\mathrm{BF}=$ breastfeed $\mathrm{EBF}=$ Exclusive breastfeeding; $\mathrm{PC}=$ peer counselor $; \mathrm{IBCLC}=$ lactation consultant 
Table 2.

Group Comparisons of Demographic, Clinical and Personal Variables at Enrollment (N=39)

\begin{tabular}{|c|c|c|c|}
\hline Variable & $\begin{array}{l}\text { Intervention group Mean (SD) or } \\
n(\%)(n=20)\end{array}$ & $\begin{array}{c}\text { Control group Mean (SD) or } \\
n(\%)(n=19)\end{array}$ & $\begin{array}{l}\text { p-value for group } \\
\text { comparison }\end{array}$ \\
\hline Mother's age & $24.3(5.2)$ & $26.6(6.6)$ & $.24^{a}$ \\
\hline \multicolumn{4}{|l|}{ Live with partner } \\
\hline Yes & $15(75.0)$ & $13(68.4)$ & $.65^{b}$ \\
\hline No & $5(25.0)$ & $6(31.6)$ & \\
\hline \multicolumn{4}{|l|}{ Employed } \\
\hline Yes & $5(25.0)$ & $6(31.6)$ & $.65^{b}$ \\
\hline No & $15(75.0)$ & $13(68.4)$ & \\
\hline \multicolumn{4}{|l|}{ Insurance } \\
\hline Yes & $7(35.0)$ & $6(31.6)$ & $.82^{b}$ \\
\hline No & $13(65.0)$ & $13(68.4)$ & \\
\hline \multicolumn{4}{|l|}{ WIC } \\
\hline Yes & $14(70.0)$ & $12(63.2)$ & $.65^{b}$ \\
\hline No & $6(30.0)$ & $7(36.8)$ & \\
\hline \multicolumn{4}{|l|}{ Mode of Delivery } \\
\hline Vaginal & $16(80.0)$ & $14(73.7)$ & $.72^{c}$ \\
\hline C-section & $4(20.0)$ & $5(26.3)$ & \\
\hline \multicolumn{4}{|c|}{ Anyone close to you breastfeed? } \\
\hline Yes & $16(80.0)$ & $14(73.7)$ & $.72^{c}$ \\
\hline No & $4(20.0)$ & $5(26.3)$ & \\
\hline Acculturation & $13.0(6.4)$ & $10.3(5.2)$ & $.16^{a}$ \\
\hline Intention to breastfeed & $13.3(1.9)$ & $11.3(4.7)$ & $.10^{a}$ \\
\hline Breastfeeding knowledge & $20.1(3.1)$ & $18.8(3.9)$ & $.25^{a}$ \\
\hline \multicolumn{4}{|l|}{${ }_{\mathrm{p}}$-value for two-sample t-test } \\
\hline
\end{tabular}

